\title{
COVID-19 outbreak and steroids administration: are patients treated for Sars-Cov-2 at risk of adrenal insufficiency?
}

\author{
C. Scaroni ${ }^{1} \cdot$ M. Armigliato $^{2} \cdot$ S. Cannavò ${ }^{3}$
}

Received: 4 April 2020 / Accepted: 8 April 2020 / Published online: 16 April 2020

(C) Italian Society of Endocrinology (SIE) 2020

In critically ill patients affected by severe acute respiratory syndrome caused by Sars-Cov-2 infection, steroids, usually methylprednisolone, are currently administered despite data on effects and lethality are contradictory. On the other hand, steroids treatment can cause symptoms of hypercortisolism, especially in patients with individual hypersensibility, or hypoadrenalism after drug withdrawal, due to hypothalamic-pituitary-adrenal (HPA) axis suppression. Adrenal insufficiency could potentially increase morbidity and mortality risk. In addition, a large part of these patients is treated by antiretroviral drugs, i.e. ritonavir (RTV), which act as potent inhibitors of the cytochrome P4503A enzymes. For this reason, the co-administration of RTV and corticosteroids may enhance the exposure to the latter ones, metabolized through the CYP450 CYP3A pathway, and can prolong their half-life, causing longer-lasting suppression of HPA axis [1]. In addition, the exposure to endocrine disruptors that decrease or modify the steroidogenic activity of adrenal glands could further impair prompt recovery of adrenal function.

Recognition of HPA impairment can be challenging. Indeed, symptoms of hypoadrenalism (fatigue, hypotension, loss of weight, nausea, vomiting, abdominal pain) are absolutely not specific and may be confused with those caused by a severe infectious disease. Moreover, especially in these specific frail patients, steroids withdrawal can also precipitate an adrenal crisis, that represents a life-threatening complication. In this scenario, adrenal insufficiency should be suspected especially when sodium concentration

S. Cannavò

cannavos@unime.it

1 Endocrine Unit, Department of Medicine, HospitalUniversity of Padua, Padua, Italy

2 Internal Medicine Unit, Mater Salutis Hospital, Legnago, VR, Italy

3 Endocrine Unit, Department of Human Pathology, University of Messina, Messina, Italy is low $(<135 \mathrm{mEq} / \mathrm{L})$, and diagnosed on the basis of morning serum cortisol levels $<80 \mathrm{nmol} / \mathrm{L}$, or cortisol peak $<550 \mathrm{nmol} / \mathrm{L}$ after stimulation with synthetic ACTH. Cortisol measurement should be postponed at least one week after the exogenous glucocorticoids tapering and withdrawal [2].

The prevalence of adrenal insufficiency following corticosteroids administration is unknown. This condition is frequently underdiagnosed, especially when patients are evaluated by non-endocrinologists. Although generally short term $(<3$ weeks) treatment does not lead to HPA axis suppression, in predisposed individuals it can occur even after short administration, as little as 5 days, and at low doses. In a recent study, adrenal insufficiency was observed in patients taking prednisolone $5 \mathrm{mg}$ per day, with mortality increase following treatment interruption [3].

Therefore, also in patients affected by COVID-19 and treated with high doses of steroids, it is necessary to perform an appropriate dose tapering. Nevertheless, if stressful events (illness, injury or surgery) occur, the dose has to be increased. Physiological glucocorticoids concentration is maintained with prednisolone $5.0-7.5 \mathrm{mg} /$ day, hydrocortisone $20 \mathrm{mg} /$ day (fractionated in $2-3$ administrations) or cortisone acetate $25 \mathrm{mg} /$ day (fractionated in 2 administrations). Because of its short half-life, hydrocortisone is generally associated with a more rapid recover of HPA function.

Unfortunately, guidelines or consensus statements suggesting how tapering steroids in this condition are lacking. For this reason, patient's management should be based on a daily monitoring of blood pressure values, weight, serum sodium levels, and clinical symptoms that indicate adrenal impairment. Finally, we remember, especially for non-endocrinologists, that serum cortisol levels have to be measured in the morning before steroids administration. 


\section{Compliance with ethical standards}

Conflict of interest The authors declare that they have no conflict of interest.

Ethical approval Not applicable.

Informed consent No informed consent is necessary for the study.

\section{References}

1. Epperla N, McKiernan F (2015) Iatrogenic Cushing's syndrome and adrenal insufficiency during concomitant therapy with ribonavir and fluticasone. SpringerPlus 4:455. https://doi.org/10.1186/ s40064-015-1218-x
2. Isidori AM, Arnaldi G, Boscaro M, Falorni A, Giordano C, Giordano R, Pivonello R, Pozza C, Sbardella E, Simeoli C, Scaroni C, Lenzi A, Italian Society of Endocrinology (2019) Towards the tailoring of glucocorticoid replacement in adrenal insufficiency: the Italian Society of Endocrinology Expert Opinion. J Endocrinol Invest. https://doi.org/10.1007/s40618-019-01146-y

3. Mebrahtu TF, Morgan AW, Keeley A, Baxter PD, Stewart PM, Pujades-Rodriguez M (2019) Dose dependency of iatrogenic glucorticoid excess and adrenal insufficiency and mortality: a cohort study in England. J Clin Endocrinol Metab 104:3757-3767. https ://doi.org/10.1210/jc.2019-00153

Publisher's Note Springer Nature remains neutral with regard to jurisdictional claims in published maps and institutional affiliations. 